\title{
Efficacy and safety of biosimilar filgrastim in primary and secondary prevention of febrile neutropenia
}

\author{
LESZEK KRAJ $^{1}$, JOANNA KRAWCZYK-LIPIEC ${ }^{1}$, JOANNA GÓRNIEWSKA ${ }^{2}$ and GRZEGORZ ORLIK ${ }^{2}$ \\ ${ }^{1}$ Department of Hematology, Oncology and Internal Diseases, The Medical University of Warsaw, \\ Warsaw 02-097; ${ }^{2}$ Accord Healthcare Polska Sp. z o.o., Warsaw 02-677, Poland
}

Received April 3, 2017; Accepted May 22, 2017

DOI: $10.3892 /$ br.2017.938

\begin{abstract}
Neutropenia and febrile neutropenia (FN) are among the most common side effects/complications of chemotherapy. The aim of the present study was to evaluate the practice of the use of biosimilar filgrastim in the primary and secondary prevention of FN, and assess its efficacy and safety. A multi-center, non-interventional epidemiological study of 170 cancer patients aged 23-82 years was conducted. Data were collected via a questionnaire completed based on medical documentation and patient examination over five chemotherapy visits. The risk of FN related to the chemotherapy protocol used was in the range of $10-20 \%$ in $>50 \%$ of the patients $(53.5 \%)$ and a majority $(74.7 \%)$ had additional $\mathrm{FN}$ risk factors. $60 \%$ of the patients received filgrastim as primary prevention of $\mathrm{FN}$, and $40 \%$ received it as secondary prevention. In $40.6 \%$ of cases, six cycles of chemotherapy were used. More than $90 \%$ of patients continued chemotherapy according to the initial recommended dose. In majority of patients, no FN was observed following the final cycle of chemotherapy. Median neutrophil count at visit 1 was $2.2 \times 10^{3} / \mu 1$ and did not fall below that level. Majority of patients ( $>70 \%$ ) performed self-injections of filgrastim, and $86.3 \%$ of patients were continuing therapy with this drug at the last visit. No treatment-related side effects were recorded. The use of biosimilar filgrastim in the primary and secondary prevention of FN allows to maintain initial chemotherapy dosage. Furthermore, the use of biosimilar filgrastim is safe and tolerable, and has a high acceptance by patients.
\end{abstract}

\section{Introduction}

Many cytotoxic drugs currently used in cancer treatment are also myelosuppressive due to their toxicity against rapidly dividing cells of the hematopoietic system. Thus, some of

Correspondence to: Dr Grzegorz Orlik, Accord Healthcare Polska Sp. z o.o., No. 7 Taśmowa Str, Warsaw 02-677, Poland

E-mail: grzegorz_orlik@accord-healthcare.com

Key words: biosimilars, chemotherapy, febrile neutropenia, filgrastim, neutropenia the most common side effects/complications of chemotherapy include injury to the hematopoietic system, mainly neutropenia or agranulocytosis. The occurrence of neutropenia, especially clinically significant neutropenia [absolute neutrophil count $\left.(\mathrm{ANC})<1 \times 10^{3} / \mu 1\right]$, is highly unfavorable and dangerous for the patient. Clinically significant neutropenia usually requires delay in the administration of the next cycle of systemic treatment and thereby reduces the dose density. This may have a negative impact on its effectiveness and worsen patient prognosis. Moreover, severe or prolonged neutropenia (ANC $<0.5 \times 10^{3} / \mu 1,>7$ days) is associated with high risk of infectious complications, which require hospitalization and aggressive treatment and may cause high mortality $(1,2)$. Clinically significant neutropenia may also be accompanied by fever in the form of febrile neutropenia (FN). According to the European Society of Medical Oncology (ESMO), FN is defined as a decrease in ANC of $<0.5 \times 10^{3} / \mu 1$ or $<1 \times 10^{3} / \mu 1$, with an expected decrease in ANC to $<0.5 \times 10^{3} / \mu 1$ within $48 \mathrm{~h}$, accompanied by fever $\left(>38.5^{\circ} \mathrm{C}\right)$ and/or clinical manifestations of sepsis (3-5). Therefore, preventing the incidence of neutropenia and FN during cytotoxic therapy within primary or secondary prevention is highly desirable.

Recombinant hematopoietic growth factors, most commonly granulocyte colony stimulating factor (G-CSF), were developed and implemented into daily clinical practice for the primary and secondary prophylaxis of neutropenia and FN. Filgrastim is one of the most widely used G-CSF currently available. It is a glycoprotein that stimulates the production and release of neutrophils from the bone marrow, resulting in a significant increase in this fraction of leukocytes in the peripheral blood within $24 \mathrm{~h}$ from drug intake, as well as a slight increase in monocytes (2-4). The use of filgrastim in patients undergoing cancer chemotherapy has been shown to reduce the incidence, severity and duration of neutropenia and FN $(3,4)$. Recommendations for clinical use of hematopoietic growth factors such as filgrastim are regulated by appropriate guidelines, including these of the ESMO and the European Organisation for Research and Treatment of Cancer $(1,3,5,6)$. In clinical practice, different preparations of filgrastim are used, including biosimilar drugs (also termed 'biosimilars' or 'follow-on biologicals'). As the name suggests, these products are biologically similar to their original drug counterparts, with similar physicochemical properties, and therefore, should possess similar efficacy and safety (7-9). 
The aim of the present study was to determine the characteristics of use of the biosimilar (follow-on) drug filgrastim in the primary and secondary prevention of $\mathrm{FN}$, as well as to evaluate its efficacy and safety.

\section{Materials and methods}

Study design. This was a multicenter, non-interventional, real-life, epidemiological study sponsored by Accord Healthcare Polska (Warsaw, Poland), which involved an electronic questionnaire. The questionnaires were completed by participating physicians (clinical oncologists) based on the analysis of patients' medical history, including laboratory tests, a targeted interview and physical examination during routine visits.

Patient characteristics. The present study ultimately included 170 cancer patients, aged 23-82 (mean 59.5 years). Demographic characteristics of included patients are presented in Table I.

Scheme of monitoring. At the first visit (visit 1), basic data (age, sex, weight and body surface area, the diagnosis of the underlying disease, disease stage and the Eastern Cooperative Oncology Group performance status) were recorded for each patient. A targeted medical interview and physical examination were also undertaken. Assessment of FN risk relating to the chosen (planned) systemic treatment protocol and the possibility of additional FN risk factors for the patient was performed. Additionally, the number of cycles of planned chemotherapy, the type of FN prevention (primary or secondary) and additional factors affecting the overall risk of FN were recorded. Finally, the overall risk of FN was evaluated. At subsequent visits (visits 2-5), the course of the G-CSF (Accofil ${ }^{\circledR}$, Accord Healthcare Ltd., North Harrow, UK) therapy was monitored, including the assessment of chemotherapy dose density, the occurrence or lack of FN following the last cycle of treatment, the continuation of G-CSF in subsequent cycles, the total G-CSF dose and whether it was self-injected, the presence of pain, and the convenience of using the drug according to patients' opinion were recorded. Hematological parameters of peripheral blood including total number of leukocytes, ANC, hemoglobin concentration and platelet count were also monitored.

Phase IV study. The study was a non-interventional post marketing study (phase IV). Accofil ${ }^{\circledR}$ was administrated in line with approved, labeled indications. Data were collected retrospectively and analyzed by statistical methods (standard descriptive statistics methods, including pie charts, bar charts and box plots). Ethical committee approval was not obtained, as personal information on study subjects was not used, processed, transferred and disclosed. Eligible patients provided informed consent before treatment. The protocol was compliant with the legal and regulatory requirements in the country.

\section{Results}

Risk of FN. The overall risk of FN, as well as risk of FN relating to the systemic treatment protocol or other factors, is
Table I. Demographic characteristics.

\begin{tabular}{lc}
\hline Sex $(\%)$ & \\
Male & 32.9 \\
Female & 67.1 \\
Age, years (min-max) & \\
Median & $62(23-82)$ \\
Body weight, kg (min-max) & \\
Median & $68(41-116)$ \\
Body surface area, ${ }^{2}$ (min-max) & \\
Median & $1.7(1.4-2.4)$ \\
Clinical stage (\%) & \\
II & 10.6 \\
III & 35.3 \\
IV & 54.1 \\
ECOG performance score (\%) & \\
0 & 14.1 \\
1 & 63.5 \\
2 & 20 \\
3 & 0.6 \\
4 & 1.8 \\
Cancer type, $n(\%)$ & \\
Breast cancers in women & $46(27.1)$ \\
Gynecological cancers & $31(18.2)$ \\
Malignant colorectal cancers & $24(14.1)$ \\
Malignant pleural and lung cancers & $23(13.5)$ \\
Blood and lymphatic tissue & $16(9.4)$ \\
Pancreatic cancers & $9(5.3)$ \\
Malignant neoplasms of male genital organs & $8(4.7)$ \\
Malignant gastric cancers & $5(2.9)$ \\
Other, <5 units $(\%)$ & $8(4.7)$ \\
\hline
\end{tabular}

ECOG, Eastern Cooperative Oncology Group.

presented in Table II. In $60 \%$ of cases, physicians estimated that the overall risk of FN was high (>20\%). The presence of additional factors that contribute to the risk of FN was found in $74.7 \%$ of cases.

Number of chemotherapy cycles. In majority of cases (40.6\%), six cycles of chemotherapy were planned; more than six cycles were planned in $28.2 \%$ of cases. Accofil ${ }^{\circledR}$ was used as primary prophylaxis in $60 \%$ of cases and as secondary prophylaxis in the remaining $40 \%$ of patients.

Continuation of therapy with Accofil ${ }^{\circledast}$. Actual continuation of therapy with Accofil ${ }^{\circledR}$ was recorded at each visit (beginning from visit 2). At visit 2, 98.2\% of patients who initiated Accofil ${ }^{\circledast}$ therapy continued, while $96.4 \%$ continued at visit 3 , $91.3 \%$ continued at visit 4 , and $86.3 \%$ continued at visit 5 . The most common reason for discontinuation of therapy was disease progression.

The dose of filgrastim taken was calculated at each of the five visits. The average (mean \pm standard deviation) dose was $220.9 \pm 84.4$ million units at visit $1 ; 222.4 \pm 87.2$ million units at 
Table II. Summary of patients' risk for FN.

\section{Type of therapy \\ Radical intention \\ Palliative}

$\mathrm{FN}$ risk associated with chemotherapy regimen used

$$
<10
$$

10-20

$>20$

Additional risk factors for $\mathrm{FN}$

Sex

Age

Previous neutropenia

Comorbidities

Previous FN

Earlier radio- or chemotherapy

Anemia

Cardiovascular diseases

Diabetes

Cancer cachexia

Internal diseases burden

Low levels of $\mathrm{Hb}$

Ischemic myocardium

No antibiotic prophylaxis

Cardiac diseases burden

Other (3 or less units)

The overall risk of FN

$<10$

10-20

$>20$
Cancer stage

$\%$

45.3

54.7

15.9

53.5

30.6

42.6

41.1

27.1

14.7

11.6

9.3

7.8

7.0

6.2

5.4

5.4

5.4

5.4

3.1

3.1

3.1

17.8

4.1

35.9

60.0

FN, febrile neutropenia; $\mathrm{Hb}$, hemoglobin.

visit $2 ; 223.9 \pm 90.3$ million units at visit $3 ; 231.6 \pm 95.7$ million units at visit 4 ; and $224.8 \pm 98.8$ million units at visit 5 . In half of the patients, the dose was $<240$ million units for all visits. Whether chemotherapy was continued at the previously recommended dose was assessed from visit 2 through the visit 5. Most patients continued chemotherapy with planned dosage (i.e., $95.3 \%$ of patients continued chemotherapy at the same dose at visit $2,94.7 \%$ at visit $3,96.2 \%$ at visit 4 , and $90.4 \%$ at visit 5). Whether FN had occurred following the previous cycle of chemotherapy was also assessed from visit 2 to visit 5 (92.9\% of patients reported no $\mathrm{FN}$ at visit $2,94.1 \%$ at visit $3,91.2 \%$ at visit 4 , and $92.6 \%$ at visit 5 ).

Convenience of injection. Patients' opinions about the convenience of the therapy were also collected. Patients were asked to assess the convenience of the use of Accofil ${ }^{\circledR}$ at each visit, using a scale of 1 to 10 , where 1 denotes the least convenient use and 10 is the most convenient. At every visit (1-5), half of the patients rated the treatment as $>8$. The average rates were: $7.4 \pm 2.1,7.7 \pm 1.8,7.9 \pm 1.8,7.9 \pm 1.7$ and $7.8 \pm 2.0$ (mean \pm standard deviation), at visits $1,2,3,4$ and 5 , respectively. The injection soreness was also evaluated. At every visit (1-5), more than half of the patients responded that the pain of the procedure was small (Fig. 1).

Hematological parameters. Hematological parameters were also assessed at each visit, with data collected prior to continuing therapy with G-CSF. At visit 1 , median neutrophil count was $2.2 \times 10^{3} / \mu 1$ and did not fall below that level at any of the five visits (i.e., median ANC were $2.7 \times 10^{3} / \mu 1,2.5 \times 10^{3} / \mu 1$, $2.4 \times 10^{3} / \mu 1$ and $2.2 \times 10^{3} / \mu 1$, at visit $2,3,4$ and 5 , respectively. Other hematological parameters are presented in Table III.

\section{Discussion}

Appropriate supportive treatment in systemic therapy of neoplastic diseases is important for maintaining the density of the chemotherapeutic dose, reducing the risk of side effects/complications and related unplanned hospitalizations, and improving patients' quality of life. Both primary and secondary FN prevention are among the most important actions in the field of supportive care during cancer chemotherapy. The ability to administer the planned dose of cytotoxic drugs on time without delaying the next cycle or a dose reduction is important for effective systemic treatment; however, this is only possible when peripheral blood parameters do not differ significantly from the reference values.

In the present study, the authors identified that the risk of neutropenia/FN associated with the applied chemotherapy was in the range of $10-20 \%$ in more than half of the patients, and that majority of patients $(>70 \%)$ presented additional risk factors for FN. Thus, a large proportion of patients were in the 'risk group' for chemotherapy-induced neutropenia and its complications. Previous systematic review including 3,494 patients demonstrated that compared to controls, the prophylactic use of G-CSF significantly reduced the relative risk (RR) of FN in patients receiving anticancer chemotherapy ( $R R$ reduction of $46 \%$ ), and reduced early mortality as well as mortality due to infectious complications (RR reductions of $40 \%$ and $45 \%$, respectively) (10). Furthermore, prophylactic use of G-CSF in these patients resulted in a significant improvement in maintaining the desired density of the cytotoxic dose (10). At the same time, a significantly higher incidence of bone and musculoskeletal pain was observed in patients receiving G-CSF compared to those without (19.6\% vs. $10.4 \%$, respectively) (10).

Clinical trials have indicated that biosimilar preparations of filgrastim have similar benefits compared to the original product, as well as similar safety and tolerability profiles. For example, in the phase III randomized trial comparing biosimilar filgrastim (EP2006) with the original product (Neupogen ${ }^{\circledR}$; Amgen Inc., Thousand Oaks, CA, USA) in the prevention of severe neutropenia in 218 patients with breast cancer receiving chemotherapy [according to the TAC (docetaxel, doxorubicin and cyclophosphamide) protocol], no significant differences in efficacy and safety were observed (11). There was also no difference between the studied preparations in the duration of severe neutropenia during the first cycle of treatment, which was the primary endpoint of the study (1.2 days for the biosimilar filgrastim and 1.2 days for the original preparation, respectively) (11). No differences 
Table III. Hematological data at each visit (V1-V5).

\begin{tabular}{lccccc}
\hline & V1 & V2 & V3 & V4 & V5 \\
\hline Hb level, median $\left(\times 10^{3} / \mu 1\right)$ & 12 & 11.7 & 11.2 & 11 & 10.9 \\
WBC level, median $\left(\times 10^{3} / \mu 1\right)$ & 5.3 & 5.6 & 5.5 & 5.3 & 5 \\
PLT level, median $\left(\times 10^{3} / \mu 1\right)$ & 246 & 232 & 222 & 223 & 211 \\
\hline
\end{tabular}

Hb, hemoglobin; PLT, platelet; WBC, white blood cell; V, visit.

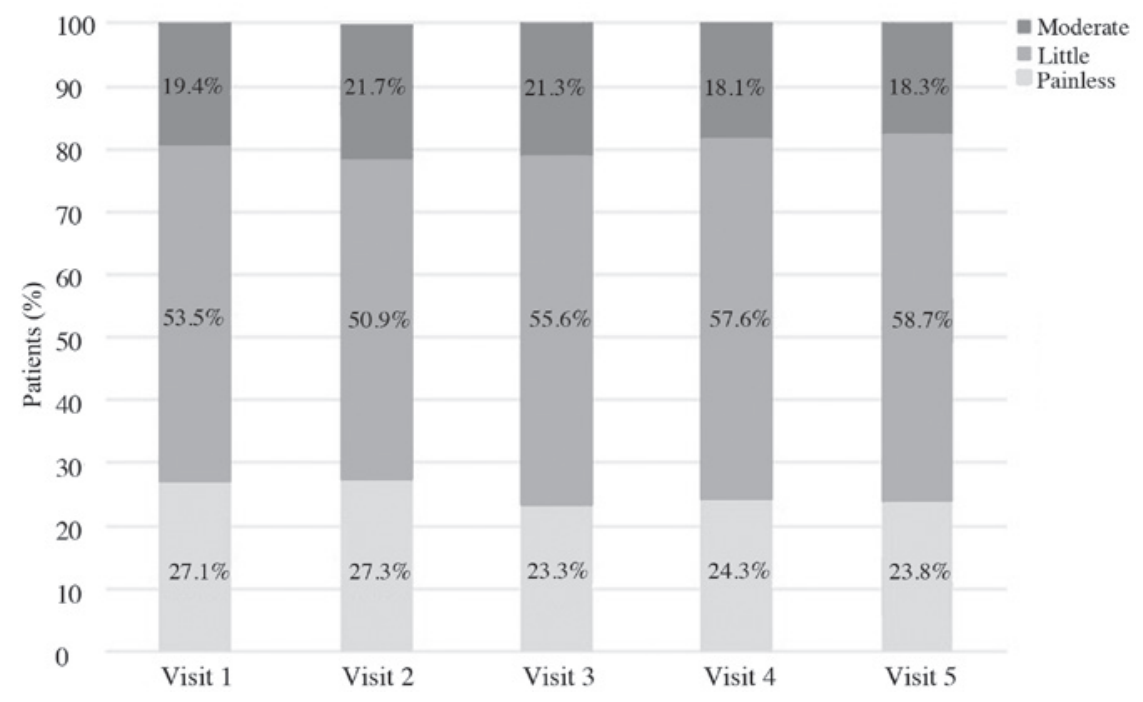

Figure 1. How patients assess the soreness of the injection procedure.

in other clinically relevant features (including the incidence of FN, the need for hospitalization due to FN, the incidence of infectious complications, depth and time of the ANC nadir and the normalization of ANC) during the first and all subsequent cycles of treatment were observed (11).

In a previous non-interventional observational study involving $>1,300$ patients undergoing chemotherapy (the HEXAFIL study), the clinical usefulness, efficacy and safety of biosimilar filgrastim was evaluated (12). A large number of patients (44.9\%) received biosimilar filgrastim for primary prevention of $\mathrm{FN}$, similar to the present study in which $60 \%$ received biosimilar filgrastim for primary prophylaxis. Approximately $90 \%$ of patients could continue chemotherapy without need for treatment modification (i.e., cytostatic dose or time of administration) (12). Complications associated with neutropenia occurred in 7.9, 6.9 and $3.9 \%$ of patients at the first, second and third treatment cycle, respectively (12). During the first cycle of chemotherapy, only $1.8 \%$ of patients experienced FN. Tolerance and safety of biosimilar filgrastim was similar to that previously known for this class of drugs; that is, bone and muscle pain were the most common treatment-related adverse events (12).

Characteristics of use and effectiveness of biosimilar filgrastim (Zarzio ${ }^{\circledR}$; Sandoz, Holzkirchen, Germany) in the prevention of chemotherapy-induced neutropenia (and FN) were also investigated in the international, multicenter, non-interventional observational (MONITOR-GCSF) study (13). In the current study involving $>1,400$ patients, the majority (72.3\%) of patients received G-CSF in primary prophylaxis (similar to the $60 \%$ recorded in our study). Based on their data, it was concluded that the clinical efficacy and safety of biosimilar filgrastim $\left(\right.$ Zarzio $\left.^{\circledR}\right)$ is comparable to historical data collected in studies on the original product (Neupogen ${ }^{\circledR}$ ) (13). Similar conclusions were drawn based in the French prospective observational multicenter study ( $n=184$ patients), where the same biosimilar filgrastim preparation (Zarzio ${ }^{\circledR}$ ) was used (14). No cases of FN were noted in the current study; however, neutropenia requiring hospitalization and/or antibiotic use occurred in 10 patients (14). Biosimilar filgrastim $\left(\right.$ Zarzio $^{\circledR}$ ) was also evaluated in a population of patients with soft tissue sarcomas receiving chemotherapy according to EI protocol (epirubicin, ifosfamide) in the NEUSAR study (15). The frequency of neutropenia of all grades and grade 4 neutropenia (ANC $<0.5 \times 10^{3} / \mu \mathrm{l}$ ) were comparable for biosimilar filgrastim and original products (Granulokine ${ }^{\circledR}$ and Neupogen ${ }^{\circledR}$; Amgen Inc.) (15). Similarly, the incidence of FN did not significantly differ with the biosimilar drug compared to the originators; the incidence was $44 \%$ for biosimilar filgrastim and $40 \%$ for the original filgrastim (12). On the basis of the present study, it was concluded that biosimilar filgrastim is effective in the prevention of $\mathrm{FN}$ and reduces the frequency of unplanned hospitalization in patients with soft tissue sarcomas (15). Comparable clinical efficacy of biosimilar filgrastim (Zarzio ${ }^{\circledR} /$ Filgrastim Hexal ${ }^{\circledR}$; Sandoz) to the original product (Neupogen ${ }^{\circledR}$ ) was also found in a non-interventional study (16), in which 77 patients with cancer were treated with 
biosimilar filgrastim and 25 were treated with the original product. In both the biosimilar filgrastim and original product treatment arms, FN only occurred in one patient (16). Only $6.5 \%$ of patients receiving biosimilar filgrastim developed neutropenia requiring chemotherapy dose reduction or its discontinuation compared with $8 \%$ of patients treated with the original product (16).

Together, the accumulating findings indicated the usefulness of biosimilar filgrastim in the primary and secondary prevention of chemotherapy-induced neutropenia/FN, as well as its clinical efficacy, safety, tolerance and acceptance by patients. In the present study, the authors evaluated a new biosimilar filgrastim, Accofil ${ }^{\circledR}$. The results are consistent with published reports, including those mentioned above. A high percentage $(>90 \%)$ of patients treated with biosimilar filgrastim (Accofil ${ }^{\circledR}$ ) could continue the chemotherapy according to plan for each of the analyzed cycles. Furthermore, a high percentage (>90\%) of patients treated with Accofil ${ }^{\circledR}$ did not experience $\mathrm{FN}$ at all cycles of chemotherapy. An added benefit of Accofil ${ }^{\circledR}$ is the ease of which it can be applied; a high percentage of patients self-injected the biosimilar filgrastim in the present study. Furthermore, Accofil ${ }^{\circledR}$ reported good tolerance and safety in the patients. Finally, both the present study and previous literature indicate that biologically similar G-CSF preparations demonstrate clinical efficacy in patients with different malignancies, including solid tumors, neoplasms of the lymphatic system, tumors of epithelial origin and soft tissues tumors.

In summary, biologically similar filgrastim (Accofil ${ }^{\circledR}$ ) used in the primary and secondary prevention of chemotherapy-induced neutropenia/FN, shows high efficiency, safety and tolerability while being readily-accepted by patients and convenient to use. By combining the current results with the data presented in the literature, it may be concluded that the efficacy and safety of Accofil ${ }^{\circledR}$ is comparable to the original filgrastim product (Neupogen ${ }^{\circledR}$ ) and to other biosimilar filgrastim preparations (Zarzio ${ }^{\circledR}$, Sandoz). Thus, Accofil ${ }^{\circledR}$ should be recommended for use in everyday clinical practice.

\section{Acknowledgements}

The present study and the preparation of the manuscript have been supported by Accord Healthcare.

\section{References}

1. Crawford J, Caserta C and Roila F: ESMO Guidelines Working Group: Hematopoietic growth factors: ESMO Clinical Practice Guidelines for the applications. Ann Oncol 21 (Suppl 5): v248-v251, 2010.

2. Dale DC: Colony-stimulating factors for the management of neutropenia in cancer patients. Drugs 62 (Suppl 1): 1-15, 2002.
3. de Naurois J, Novitzky-Basso I, Gill MJ, Marti FM, Cullen MH and Roila F; ESMO Guidelines Working Group: Management of febrile neutropenia: ESMO Clinical Practice Guidelines. Ann Oncol 21 (Suppl 5): v252-v256, 2010.

4. European Commission: Community register of medicinal products for human use: Accofil. http://ec.europa.eu/health/ documents/community-register/html/h946.htm. Accessed Feb 11, 2017.

5. Greil R, Psenak O and Roila F; ESMO Guidelines Working Group: Hematopoietic growth factors: ESMO recommendations for the applications. Ann Oncol 19 (Suppl 2): ii116-ii118, 2008.

6. Aapro MS, Bohlius J, Cameron DA, Dal Lago L, Donnelly JP, Kearney N, Lyman GH, Pettengell R, Tjan-Heijnen VC, Walewski J, et al: European Organisation for Research and Treatment of Cancer: 2010 update of EORTC guidelines for the use of granulocyte-colony stimulating factor to reduce the incidence of chemotherapy-induced febrile neutropenia in adult patients with lymphoproliferative disorders and solid tumours. Eur J Cancer 47: 8-32, 2011.

7. Weise M, Bielsky MC, De Smet K, Ehmann F, Ekman N, Narayanan G, Heim HK, Heinonen E, Ho K, Thorpe R, et al: Biosimilars-why terminology matters. Nat Biotechnol 29: 690-693, 2011.

8. Dranitsaris G, Amir E and Dorward K: Biosimilars of biological drug therapies: Regulatory, clinical and commercial considerations. Drugs 71: 1527-1536, 2011.

9. Gottlieb S: Biosimilars: Policy, clinical, and regulatory considerations. Am J Health Syst Pharm 65 (Suppl 6): S2-S8, 2008.

10. Kuderer NM, Dale DC, Crawford J and Lyman GH: Impact of primary prophylaxis with granulocyte colony-stimulating factor on febrile neutropenia and mortality in adult cancer patients receiving chemotherapy: A systematic review. J Clin Oncol 25: 3158-3167, 2007.

11. Blackwell K, Semiglazov V, Krasnozhon D, Davidenko I, Nelyubina L, Nakov R, Stiegler G, Singh P, Schwebig A, Kramer S, et al: Comparison of EP2006, a filgrastim biosimilar, to the reference: A phase III, randomized, double-blind clinical study in the prevention of severe neutropenia in patients with breast cancer receiving myelosuppressive chemotherapy. Ann Oncol 26: 1948-1953, 2015.

12. Tesch H, Ulshöfer T, Vehling-Kaiser U, Ottillinger B, Bulenda D and Turner M: Prevention and treatment of chemotherapy-induced neutropenia with the biosimilar filgrastim: A non-interventional observational study of clinical practice patterns. Oncol Res Treat 38: 146-152, 2015.

13. [Erratum] Gascón P, Aapro M, Ludwig H, Bokemeyer C, Boccadoro M, Turner M, Denhaerynck K, MacDonald K and Abraham I: Treatment patterns and outcomes in the prophylaxis of chemotherapy-induced (febrile) neutropenia with biosimilar filgrastim (the MONITOR-GCSF study). Support Care Cancer 24: 911-925, 2016.

14. Nahon S, Rastkhah M, Ben Abdelghani M, Soumoudronga RF, Gasnereau I and Labourey JL: Zarzio ${ }^{\circledR}$, biosimilar of filgrastim, in prophylaxis of chemotherapy-induced neutropenia in routine practice: A French prospective multicentric study. Support Care Cancer 24: 1991-1998, 2016.

15. Bongiovanni A, Monti M, Foca F, Recine F, Riva N, Di Iorio V, Liverani C, De Vita A, Miserocchi G, Mercatali L, et al: Recombinant granulocyte colony-stimulating factor (rG-CSF) in the management of neutropenia induced by anthracyclines and ifosfamide in patients with soft tissue sarcomas (NEUSAR). Support Care Cancer 25: 111-117, 2017.

16. Verpoort K and Möhler TM: A non-interventional study of biosimilar granulocyte colony-stimulating factor as prophylaxis for chemotherapy-induced neutropenia in a community oncology centre. Ther Adv Med Oncol 4: 289-293, 2012. 